\title{
Transcranial ultrasound - clinical applications in cerebral ischaemia
}

\author{
C.R. Levi, Staff Neurologist and Senior Lecturer, and C. Selmes, Research \\ Scientist, Clinical Neuroscience Program, Hunter Medical Research Institute, \\ John Hunter Hospital, Newcastle; and B.R. Chambers, Director, Neurosonology, \\ Austin and Repatriation Medical Centre and Head, Ultrasound Research \\ Division, National Stroke Research Institute, Melbourne
}

To view videos of cerebral ultrasound scans click here

\section{SYNOPSIS}

Transcranial ultrasound can rapidly and non-invasively image blood flow in the major basal intracranial arteries. Its accuracy makes it acceptable for use in screening for haemodynamically significant intracranial stenoses or vessel occlusions. Although it has a relatively limited field of view and is not technically feasible in approximately $10 \%$ of cases, the information obtained is becoming increasingly relevant to therapeutic decision-making in the prevention and management of stroke. Transcranial Doppler ultrasound or transcranial colour-coded duplex have the advantages of relatively low cost, ease of repeatability, and excellent safety and tolerability, but they provide inferior spatial and anatomical detail in comparison to angiographic techniques.

Index words: stroke, magnetic resonance angiography, angiography.

(Aust Prescr 2001;24:137-40)

\section{Introduction}

Every year approximately 40000 Australians have a stroke and by 2020 the number of cases is expected to rise by $60 \%{ }^{1}$ Stroke is a heterogeneous disorder with many clinical manifestations and aetiologies. The most common cause is occlusion of a large artery, resulting from or associated with thrombosis and/or artery-to-artery embolism. ${ }^{2}$ Angiographic and sonographic imaging of intracranial vessels within six hours of the onset of an ischaemic stroke shows large artery occlusion in up to $70 \%$ of patients. ${ }^{3,4}$ These occlusions are most commonly caused by emboli and suggest one or more sources in a proximal large artery, the aorta or the heart. In up to $66 \%$ of these patients the likely embolic source is atheromatous disease of the extracranial or intracranial carotid arteries, vertebral arteries, basilar artery or middle cerebral arteries. Approximately 5-8\% of clinically relevant large artery disease in the anterior circulation (carotid, middle cerebral and anterior cerebral arteries) is located intracranially. ${ }^{2}$ This increases to approximately $30 \%$ in the posterior circulation (vertebro-basilar/posterior cerebral arteries). ${ }^{2,5}$ Although intracranial large artery occlusive disease is a considerably less common cause of artery-to-artery embolism than extracranial disease in the Caucasian population, the absence of a defined embolic source in the extracranial vessels or the heart should prompt evaluation of the intracranial vessels.

\section{Transcranial ultrasound (Fig. 1)}

The use of Doppler ultrasound to view intracranial basal artery blood flow was first described in $1982 .{ }^{6}$ Subsequent advances in ultrasound technology have seen the use of combination Doppler blood flow imaging and B mode tissue imaging, so-called transcranial colour-coded duplex. ${ }^{7}$ This more precisely identifies vessels and, if required, the direction of flow. Doppler beam angle correction gives a more accurate estimate of blood flow velocity in areas of arterial tortuosity.

Fig. 1

Schematic image of the sectional planes for the temporal and foramen magnum acoustic windows

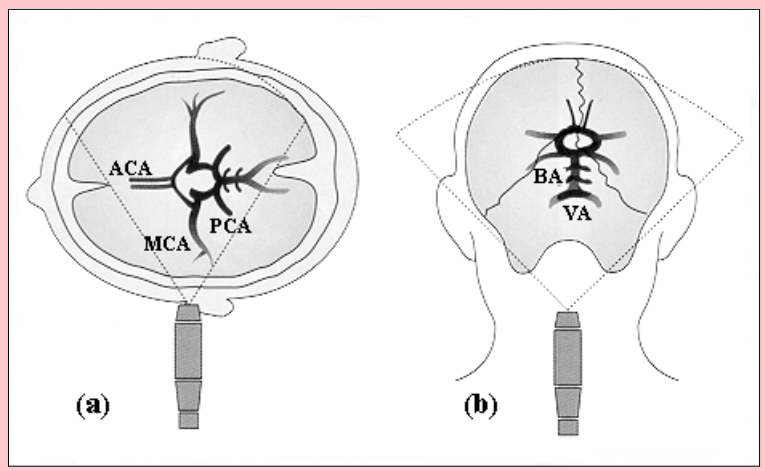

The middle cerebral artery (MCA), anterior cerebral artery (ACA) and posterior cerebral artery (PCA) are imaged via the temporal window (a). The intracranial vertebral arteries (VA) and the basilar artery (BA) are imaged via the foramen magnum window (b).

From Bartels E. Makropathologie. Color-Coded Duplex Ultrasonography of the Cerebral Vessels. Stuttgart, New York: Schattauer 1999. 
Fig. 2

Colour-coded duplex scanning of normal intracranial vessels

Images are best seen in the web (HTML)

version of this article
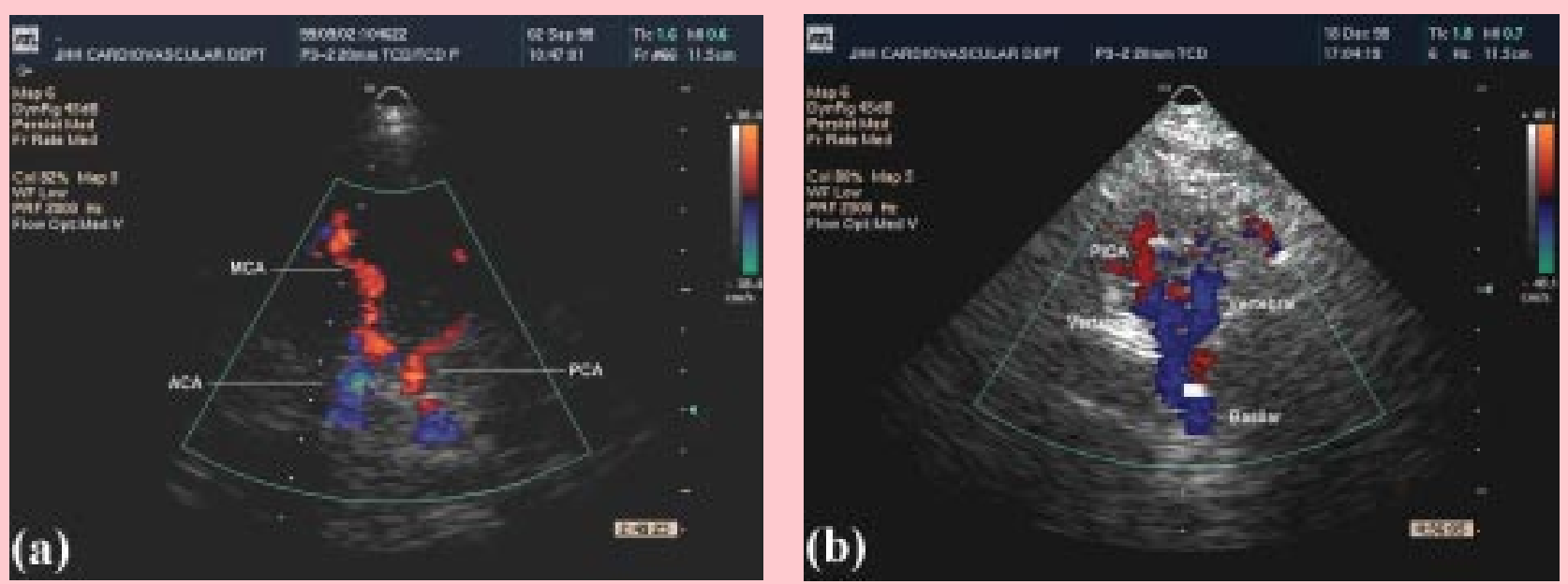

Flow direction is colour coded with flow directed towards the transducer coded red, and flow away coded blue. Velocity is colour coded within the red/blue spectrum. Regions of focal velocity increase show as brighter zones.

(a) Segments of Circle of Willis in one cerebral hemisphere as imaged via the temporal acoustic window. This window shows the M1 and M2 segments of the middle cerebral artery (MCA), the P1 and P2 segments of the posterior cerebral artery (PCA) and the A1 segment of the anterior cerebral artery (ACA). (b) Intracranial vertebral arteries and basilar artery to the mid portion as imaged via the foramen magnum acoustic window. The distal one-third of basilar insonation is often limited and identification of anterior and posterior inferior cerebellar arteries (PICA) is variable.
Transcranial Doppler ultrasound, and more recently transcranial colour-coded duplex, have been used for a variety of clinical purposes:

- screening for vasospasm following subarachnoid haemorrhage

- screening for intracranial vessel stenoses and occlusions in ischaemic stroke or transient cerebral ischaemia

- monitoring changes in intracranial haemodynamics and monitoring for emboli during carotid endarterectomy

- monitoring cerebral perfusion in the neurological intensive care setting.

Examples of transcranial colour-coded duplex studies of a normal circle of Willis and a normal intracranial vertebrobasilar system are shown in Figure 2. Figure 3 shows an example of a flow map and Doppler spectrum imaged in a patient with stenosis of the anterior cerebral artery.

When considering the use of transcranial Doppler ultrasound or transcranial colour-coded duplex as a screening test for occlusive disease in large intracranial arteries, the following issues need to be considered:

- accuracy - sensitivity and specificity

- feasibility - safety and tolerability, availability, costeffectiveness

- reliability - intra- and interobserver variability in scanning and reporting.

\section{Accuracy}

Both transcranial Doppler ultrasound and transcranial colourcoded duplex provide a relatively limited view of the basal cerebral arteries (Fig. 1). The vessels that are visible on transcranial Doppler ultrasound do, however, comprise the more common sites for the development of intracranial large artery occlusive disease. It is also important to appreciate that finding a stenosis using Doppler depends on the detection of a haemodynamic disturbance of sufficient significance to produce an unequivocal elevation in blood flow velocity. In general, this requires the presence of a diameter narrowing of approximately $50 \%$ or more (equivalent to a cross-sectional area narrowing of approximately $\geq 75 \%$ ). Although somewhat arbitrary, these cut-offs are of clinical relevance as it is not until diameter narrowing exceeds $50 \%$ that reduction in blood flow occurs. Studies of the accuracy of transcranial Doppler ultrasound or transcranial colour-coded duplex have tended, therefore, to use $50 \%$ or greater diameter narrowing as the threshold of abnormality.

The accuracy of both transcranial Doppler ultrasound and transcranial colour-coded duplex in the detection of intracranial stenoses or occlusions has been investigated in a number of comparative studies. However, there are relatively few studies assessing the accuracy of transcranial colour-coded duplex. More recently, a number of smaller studies have found that advances in instrumentation and the use of echo-enhancing agents can improve image quality and the imaging of vessel segments that are poorly seen with standard techniques. ${ }^{8,9}$ These advances are likely to result in improvements in both the feasibility and accuracy of transcranial colour-coded duplex.

In general, the accuracy of transcranial Doppler ultrasound and transcranial colour-coded duplex in the detection of intracranial occlusive disease can be considered acceptable 
Fig. 3

Colour flow map and pulse wave Doppler spectrum of a stenosis

Images are best seen in the web (HTML) version of this article

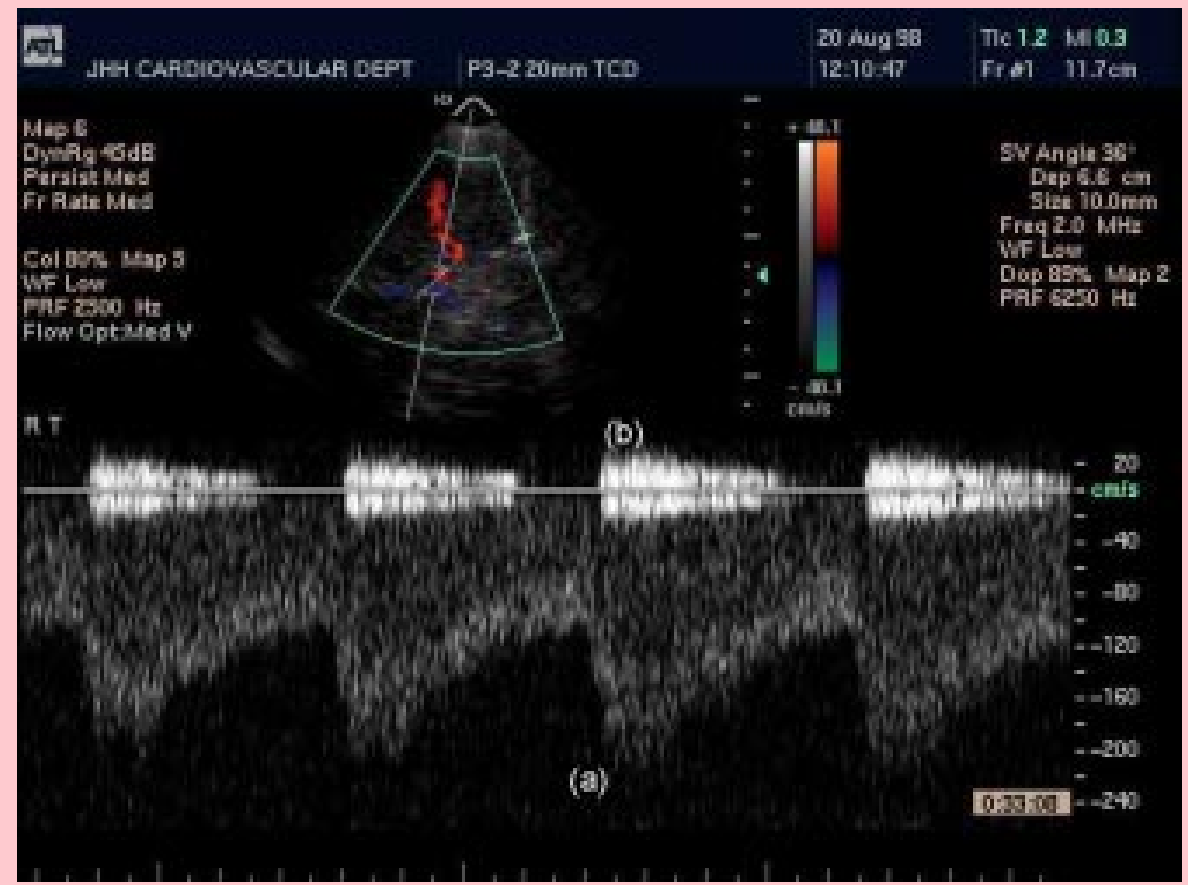

Colour-coded duplex scanning via the right temporal acoustic window shows a focal region of increase in colour intensity in the A1 section of the anterior cerebral artery. This is accompanied by an increase in (a) flow velocity (peak systolic velocity $224 \mathrm{~cm} / \mathrm{sec}$ ), and (b) turbulence (high intensity, low velocity signals). Other general criteria for haemodynamically significant stenoses include circumscribed local increase in flow velocity, and left/right difference in flow velocity (in the above example, left ACA peak systolic velocity was $86 \mathrm{~cm} / \mathrm{sec}$ ).

when performed as 'opportunistic' screening by sonographers and clinicians experienced in its use.

\section{Comparison with angiography}

When compared to contrast angiography, transcranial Doppler ultrasound accuracy varies with the technical difficulty typically encountered in imaging any particular segment. The middle cerebral artery stem (M1 segment) is relatively easy to study, so ultrasound has a sensitivity and specificity of $90-99 \%$ for finding a stenosis or an occlusion. ${ }^{10}$ For the more difficult to image intracranial (V4) segment of the vertebral arteries and the basilar artery, ultrasound has a sensitivity of $70-80 \%$ and a specificity of $90-99 \% .^{10,11}$

The accuracy of transcranial colour-coded duplex ultrasound is similar to that of contrast angiography. In a study of 310 patients, transcranial colour-coded duplex correctly identified 31 who had a stenosis of $50 \%$ or greater (confirmed by digital subtraction X-ray angiography). ${ }^{12}$ As all the stenoses were correctly diagnosed as true positives the sensitivity is $100 \%$, but the specificity is $99 \%$ because one artery with a stenosis of less than $50 \%$ was incorrectly classified as having a stenosis of more than $50 \%$ (false positive).

\section{Comparison with magnetic resonance angiography (MRA)}

Intracranial MRA has a reasonable accuracy in comparison to contrast angiography for intracranial stenoses. ${ }^{13}$ The studies to date also suggest a good correlation between MRA and transcranial colour-coded duplex for imaging a stenosis or occlusion. In the anterior circulation arterial segments, transcranial colour-coded duplex has an estimated sensitivity of $75 \%$ and specificity of $99 \%$, while in the posterior circulation, the sensitivity is $88 \%$ and the specificity $99 \% .^{14}$

\section{Feasibility}

The main limitation of transcranial ultrasound is the inadequacy of the acoustic window through either the temporal bone or the foramen magnum. The temporal window is more likely to be inadequate for ultrasound in women and the elderly. Up to $10 \%$ of patients undergoing transcranial Doppler ultrasound and up to $20 \%$ undergoing transcranial colour-coded duplex have inadequate acoustic windows for an optimal study. These proportions can be reduced to less than $5 \%$ with the use of intravenous transpulmonary echo-contrast drugs, however, these drugs increase the complexity and cost of the investigation.

Transcranial Doppler ultrasound and transcranial colour-coded duplex have no recognised adverse effects, a potential advantage over X-ray angiography which carries a risk of contrast allergy and stroke ${ }^{15}$, or computerised tomographic angiography that uses ionizing radiation and also requires intravenous contrast media. The ultrasound studies are well tolerated, a potential advantage over MRA which sometimes triggers claustrophobia, necessitating the use of intravenous sedation.

Transcranial Doppler ultrasound and transcranial colour-coded duplex cost less than angiography, and the portability of the equipment allows the examination to be performed at the patients' bedside and at repeated intervals if necessary. 
Studies assessing the cost-effectiveness of transcranial Doppler ultrasound or transcranial colour-coded duplex in cerebrovascular disease have not been reported.

\section{Reliability}

One of the potential limitations in all diagnostic vascular ultrasound is that a high level of technical and procedural skill is required to obtain the best quality images. In addition, a clear understanding of the clinical question being asked often enhances the detail obtained by the sonographer. Both these issues are particularly pertinent to transcranial ultrasound. While there are established criteria ${ }^{16}$ for the diagnosis of intracranial stenoses and occlusion using either transcranial Doppler ultrasound or transcranial colour-coded duplex, there are few studies evaluating intra- or interobserver variations in reporting.

\section{Imaging influences management}

Advances in brain imaging techniques now allow therapeutic decisions about the acute management and prevention of stroke to be made with a detailed understanding of the nature and severity of the underlying arterial pathology. ${ }^{17}$ For example, in intracranial occlusive disease, the finding of a middle cerebral artery or basilar artery occlusion in a patient with an acute stroke, is an indication for the use of thrombolytic therapy (in appropriately resourced centres). ${ }^{18,19}$ Conversely, the finding of a high grade intracranial stenosis in the artery supplying a recently ischaemic region of the brain, should prompt the clinician to consider prescribing anticoagulation rather than antiplatelet therapy for secondary prevention, as present evidence suggests anticoagulation has a therapeutic advantage. Without imaging to assess the arterial pathology, the most appropriate evidence-based intervention cannot be identified.

\section{Conclusion}

The major clinical application of transcranial ultrasound is as a specific investigation for intracranial occlusive disease when used in conjunction with ultrasonic studies of the extracranial cerebral vasculature and computerised tomographic scanning of the brain. This information can be used to guide the choice of treatment for preventing and managing strokes. Advances in instrumentation and the application of echo-contrast drugs are likely to improve the accuracy and reliability of transcranial ultrasound techniques.

E-mail: christopher.levi@hunter.health.nsw.gov.au

\section{NOTE}

To view videos of cerebral ultrasound scans click here

\section{REFERENCES}

1. National Health and Medical Research Council. Clinical practice guidelines. Prevention of stroke. The role of anticoagulants, antiplatelet agents and carotid endarterectomy. Canberra: NHMRC; 1996.

2. Mohr JP, Sacco RL. Classification of ischaemic strokes. In: Barnett HJM, editor. Stroke: pathophysiology, diagnosis and management. 2 nd ed. New York: Churchill Livingstone; 1992. p. 271-83.

3. Pessin MS, Adams HP, Adams RJ, Fisher M, Furlan AJ, Hacke W, et al. American Heart Association Prevention Conference. IV. Prevention and rehabilitation of stroke. Acute interventions. Stroke 1997;28:1518-21.

4. Alexandrov AV, Demchuk AM, Wein TH, Grotta JC. Yield of transcranial Doppler in acute cerebral ischemia. Stroke 1999;30:1604-9.

5. Caplan LR. Brain embolism, revisited. Neurology 1993;43:1281-7.

6. Aaslid R, Markwalder TM, Nornes H. Noninvasive transcranial Doppler ultrasound recording of flow velocity in basal cerebral arteries. J Neurosurg 1982;57:769-74.

7. Bogdahn U, Becker G, Winkler J, Greiner K, Perez J, Meurers B. Transcranial colour-coded real-time sonography in adults. Stroke 1990;21:1680-8.

8. Nabavi DG, Droste DW, Kemeny V, Schulte-Altedorneburg G, Weber S, Ringelstein EB. Potential and limitations of echocontrast-enhanced ultrasonography in acute stroke patients: a pilot study. Stroke 1998;29: 949-54.

9. Schlief R. Galactose-based echo contrast agents in diagnostic ultrasound. In: Becker G, Bogdahn U, Schlachetzki F. Echoenhancers and transcranial color duplex sonography. Berlin: Blackwell Science; 1998. p. 157-67.

10. Demchuk AM, Christou I, Wein TH, Felberg RA, Malkoff M, Grotta JC, et al. Accuracy and criteria for localizing arterial occlusion with transcranial Doppler. J Neuroimaging 2000;10:1-12.

11. de Bray JM, Missoum A, Dubas F, Emile J, Lhoste P. Detection of vertebrobasilar intracranial stenoses: transcranial Doppler sonography versus angiography. J Ultrasound Med 1997:16;213-8.

12. Baumgartner RW, Mattle HP, Schroth G. Assessment of $\geq 50 \%$ and $<50 \%$ intracranial stenoses by transcranial color-coded duplex sonography. Stroke 1999;30:87-92.

13. Oelerich M, Lentschig MG, Zunker P, Reimer P, Rummeny EJ, Schuierer G. Intracranial vascular stenosis and occlusion: comparison of 3D time-of-flight and 3D phase-contrast MR angiography. Neuroradiology 1998;40:567-73.

14. Levi CR, Gacs Z, Schwartz R, Hudson P, Hardy D, Bull N, et al. The accuracy of intracranial large artery occlusive disease assessment using transcranial colour coded duplex sonography. Stroke 2000;31:336.

15. Hankey GJ, Warlow CP, Sellar RJ. Cerebral angiographic risk in mild cerebrovascular disease. Stroke 1990;21:209-22.

16. Wechsler LR. Cerebrovascular disease. In: Babikian VL, Wechsler LR, editors. Transcranial Doppler ultrasonography. 2nd ed. Boston, MA: Butterworth-Heinemann; 1999. p. 91-108.

17. Davis SM, Donnan GA, Grotta JC, Hacke W. Thrombolytic therapy in acute ischemic stroke. In: Interventional therapy in acute stroke. Malden, MA: Blackwell Science; 1998. p. 87-101.

18. Furlan A, Higashida R, Wechsler L, Gent M, Rowley H, Kase C, et al. Intra-arterial prourokinase for acute ischemic stroke. The PROACT II study: a randomized controlled trial. Prolyse in acute cerebral thromboembolism. JAMA 1999;282:2003-11.

19. European Stroke Initiative recommendations for stroke management. European Stroke Council, European Neurological Society and European Federation of Neurological Societies. Cerebrovasc Dis 2000;10:335-51.

\section{Conflict of interest: none declared}

\section{Self-test questions}

The following statements are either true or false (answers on page 158)

1. Patients with an allergy to contrast media should not have colour-coded duplex scanning.

2. Occlusions of large cerebral arteries are usually caused by emboli from elsewhere in the vascular system. 